\title{
Age-varying associations between e-cigarette use and peer use, household use, and exposure to e-cigarette commercials among alternative high school students in Southern California
}

\author{
Ndifreke Etim', James Pike ${ }^{1}$, Bin Xie ${ }^{1}$
}

\begin{abstract}
INTRODUCTION Electronic cigarettes (e-cigarettes) have rapidly become the most commonly used tobacco product among youth in the United States. Exposure to advertising, peer use, and household use, increases the risk of current e-cigarette use; however, the influence of these factors may be dynamic across adolescence. The aim of this study is to examine the age-varying associations between e-cigarette use and peer use, household use, and exposure to e-cigarette commercials among alternative high school students in Southern California.

METHODS Using data previously collected for a tobacco marketing study, we examine the age-varying associations of current e-cigarette use and three risk factors (peer use, exposure to commercials, and household use) across ages 15 to 20 years using time-varying effect modeling (TVEM). Analyses include three waves of data from alternative high school students ( $\mathrm{N}=1060$ students; 2036 observations).

RESULTS The probability of e-cigarette use gradually increased over the age of 15 years and then decreased by the age of 17 years for females and after the age of 18 years for males. Significant gender differences were observed between the ages of 17 and 19.5 years. Peer e-cigarette use was associated with higher odds of current e-cigarette use across all ages for females and after the age of 16 years for males. Exposure to e-cigarette commercials increased current use significantly for males between the ages of 16 and 18 years. Household use increased the odds of current use of e-cigarettes between the ages of 17 and 19 years for males and under 16.5 and over 18 years for females.

concLusions The findings highlight the utility of TVEM in understanding the risk factors of e-cigarette use and suggest that these factors are dynamic across adolescence.
\end{abstract}

\section{AFFILIATION}

1 School of Community and Global Health, Claremont Graduate University,

Claremont, United States

CORRESPONDENCE TO Ndifreke Etim. School of Community and Global Health, Claremont Graduate University, 675 West Foothill Boulevard, Claremont, CA 91711, United States. E-mail: ndifreke.etim@cgu.edu

KEYWORDS

adolescents, electronic cigarette, peer influence

Received: 30 July 2019 Revised: 5 December 2019 Accepted: 9 January 2020

\section{INTRODUCTION}

Electronic cigarettes (e-cigarettes) are the most common nicotine product used by adolescents and young adults in the United States ${ }^{1}$. In 2018, 20.8\% of high school students and $4.9 \%$ of middle school students reported e-cigarette use in the last 30 days $^{2}$. Research on the long-term health consequences of e-cigarette use is limited. However, systematic reviews have classified e-cigarettes as a hazardous product ${ }^{3}$. Moreover, meta-analyses suggest that youth who use e-cigarettes are more likely to use traditional cigarettes $^{4}$, which are estimated to cause 0.48 million premature deaths and 12.7 million medical conditions each year ${ }^{5}$.

Alternative high school students are at a disproportionally higher risk for substance abuse than traditional high school students ${ }^{6,7}$. Unfortunately, this group of students is consistently excluded from important national surveys such as the Youth Risk Behavior Survey (YRBS), and have been only 
included in 1998, when the survey found that $64.1 \%$ of students at alternative high schools had smoked cigarettes in the last 30 days $^{8}$. Recent studies of alternative high schools have reported prevalence of cigarette use in the last 30 days between $38.7 \%$ to $56.3 \%{ }^{9,10}$, compared to $8.1 \%$ among traditional high school students ${ }^{11}$. Given that this population is at high risk for substance use, an understanding of the dynamics of social network predictors for alternative nicotine products, such as e-cigarettes, may help to inform future research and guide the development of interventions to prevent e-cigarette use among at-risk adolescents.

Prior research indicates that most smokers initiate tobacco use during adolescence, when normative and deviant behaviors are learned from elements of their social environment, including family, peers, and media $^{12,13}$. The dynamics of smoking in social networks (i.e. closest friends, family, any household member) suggests that a social environment supportive of e-cigarette use or use of combustible tobacco is associated with greater susceptibility to and increased likelihood of using e-cigarettes or smoking ${ }^{14,15}$. The use of e-cigarettes by someone else in the home is associated with higher odds of current or ever use of e-cigarettes, and an increase in the susceptibility to cigarette smoking, irrespective of e-cigarette smoking status ${ }^{15,16}$. A growing body of evidence suggests that adolescents with family and friends who use e-cigarettes are significantly more likely to be e-cigarette users ${ }^{17,18}$. Studies have also established an association between exposure to e-cigarette advertising and e-cigarette initiation and use, among youth $^{19,20}$. It has even been suggested that exposure to e-cigarettes may be more effective at attracting new users than encouraging established smokers to switch products ${ }^{21}$. Taken together, the supportiveness of the social environment towards e-cigarettes may be shaping lifelong behaviors among adolescents at a time when they are particularly susceptible to outside influences.

To better understand these effects, it is critical to examine whether the power of these influences changes as one ages. Previous studies on tobacco use have shown variations in these predictors across different developmental stages ${ }^{18,21}$. Villanti et al. ${ }^{22}$ found that the effect of peer smoking decreases from early adolescence to middle adolescence, while the effect of family smoking is static across each developmental stage. These researchers also found that exposure to tobacco-related media is associated with increased smoking in both early and middle adolescence. Liao et al. ${ }^{18}$ found that the effect of peer cigarette use was higher during junior high school than senior high school. The objective of the current study was to expand upon prior research by examining the age-varying associations between e-cigarette use and peer use, household use, and exposure to e-cigarette commercials among alternative high school students in Southern California, between the ages of 15 and 20 years.

\section{METHODS}

\section{Sampling}

Participants were recruited from alternative high schools that had at least 100 students and were within 100 miles of the program offices in Claremont, California. Data provided by the California Department of Education were used to identify 183 alternative high schools that met these criteria. Following approval by the Institutional Review Board, administrators at the 183 schools were contacted in a randomly selected order. The first 29 schools that agreed to participate were enrolled in the study on a first-come, first-served basis. Between 14 October 2014 and 18 May 2015, interest forms were distributed to 6870 students from the 29 schools. A total of 2726 students returned a completed form. Program staff contacted interested students and their parents or guardians. Each participant provided written consent. Parental consent was obtained for individuals under the age of 18 years. After acquiring consent, staff members arranged a date and time for each student to complete a web-based 90-minute survey.

A total of 1060 students took part in the initial assessment, which was a $15.4 \%$ response rate (1060/6870). Each student received a $\$ 45$ gift card to compensate them for their time. At the 1-year follow-up assessment, $87.1 \%$ of the original cohort (923/1060) completed a web-based (892) or phone (31) survey. Each student that completed a survey received a $\$ 50$ gift card. The 137 students who did not complete a follow-up assessment had failed to respond to repeated contact attempts $(n=128)$, withdrew from the study $(n=8)$, or were incarcerated $(\mathrm{n}=1)$. 
At the 2-year follow-up, $81.0 \%$ of the initial cohort (859/1060) completed a survey. A total of 832 students completed a web-based survey, while 27 students completed a computer-assisted telephone interview. Each participant that completed an assessment received a $\$ 100$ gift card. The 201 participants that did not complete an assessment had failed to respond to repeated contact attempts $(\mathrm{n}=187)$, withdrew from the study $(\mathrm{n}=9)$, had died $(n=3)$, were incarcerated $(n=1)$ or were deployed overseas after enrolling in the military $(n=1)$. During the 2-year follow-up assessment, an effort was made to contact each of the 128 students who had failed to respond to repeated contact attempts during the 1 -year follow-up assessment. None of the students returned messages delivered by email, text, phone or through social media.

Data were collected on the variables of focus in the present investigation as well as a number of additional variables beyond the scope of this study. These variables have been described in other publications ${ }^{23,24}$. The present focus on age-varying associations does not overlap with other work on these data.

Demographics such as sex, ethnicity and birthdate were assessed for each student. Birthdate was used to calculate the age of the student at the time of assessment, with a mean age of approximately 17.46 $(\mathrm{SD}=0.88)$ years at baseline. Sex was recorded according to self-report, with $50.3 \%$ reporting being male and $49.7 \%$ female. Ethnicity was recorded according to self-report, with $75.2 \%$ of the baseline sample reporting being Hispanic and $24.8 \%$ not Hispanic.

\section{Measures}

E-cigarette use in the past 30 days

To quantify recent e-cigarette use, students were asked: 'During the past 30 days, on how many days did you use electronic cigarettes, vaporizers, or vape pens?'. Response options included: $0,1-2,3-5,6-9$, $10-19,20-29$, or 30 days. Recent use was coded as ' 0 ' if no use was reported in the past 30 days, and ' 1 ' if use was reported.

\section{Exposure to e-cigarette commercials}

Two initial questions asked participants if they had ever seen an e-cigarette commercial on television or the internet. Frequency of exposure to e-cigarette commercials in the last 6 months was assessed by asking participants: 'About how often did you see an electronic cigarette commercial in the last six months?'. Response options included: never, less than once a month, once a month, $2-3$ times a month, once a week, 2-6 times a week, or every day.

\section{Household use of e-cigarettes}

A single item was used to assess the household use of e-cigarettes. Students were asked: 'Does anyone who lives with you now use electronic cigarettes, vaporizers, or vape pens?'. Participants could respond with a yes or no.

\section{Peer use of e-cigarettes}

Information on peer use of e-cigarettes was obtained by asking participants: 'How many of your four closest friends use electronic cigarettes, vaporizers, or vape pens?'. Response options included: none, one, two, three, four, or not sure. Participants who replied 'not sure' were recoded as 'missing'.

\section{Analysis}

For the current analysis, data from the three waves were combined for all participants. Only participants aged 15-20 years were included in this analysis due to low frequencies below 15 or over 20 years of age. This resulted in an analytic sample size of 2034 observations. Time-varying effect modeling (TVEM) uses all available data and accounts for within-subject correlation while addressing challenges related to unequal observations such as an unbalanced number of assessments and missing data ${ }^{25}$. For this analysis, observations from each participant were missing if the participants were below 15 or over 20 years of age, if the participants had dropped out of the study, or if measures of interest were not provided. Chi-squared tests or Fisher's exact test was used to examine differences in participants with missing data due to dropping out or planned absence due to age. Males were more likely to have dropped out of the study compared to females $(p=0.005)$. There was no difference in baseline e-cigarette use $(p=0.660)$, exposure to e-cigarette commercials $(\mathrm{p}=0.766)$, peer e-cigarette use $(p=0.283)$ and household e-cigarette use $(p=0.731)$ between both groups.

The TVEM SAS Macro with p-spline smoothing 
was used in SAS v9.4 to fit a logistic age-varying effect mode $^{26,27}$. TVEM was used to estimate the prevalence rate of e-cigarette use as a function of age, and the age-varying association between e-cigarette use and each of the following variables: household use of e-cigarettes, peer use of e-cigarettes, and exposure to e-cigarette commercials. The model assumed that this change occurred smoothly over time, but did not make any parametric assumptions (e.g. linear or quadratic) about the change in effects ${ }^{27-29}$. Within each assessment year, all variables in the model were measured at the same time point; hence, crosssectional relationships were estimated by the model.

First, an intercept-only model was used to estimate the rate of e-cigarette use as a function of age. Second, a model including sex as a covariate was used to examine subgroup differences in the age-varying prevalence of e-cigarette use. Third, TVEM models were run for the age-varying associations between time-varying predictors and outcomes overall. Finally, since sex was significantly related to the age-varying rate of e-cigarette use $(\mathrm{p}<0.05)$, TVEM models were estimated separately for males and females. All three predictors were included together in the model, so that the other two served as time-varying covariates. Ethnicity (Hispanic vs not Hispanic) was included as a time-invariant covariate in all models.

\section{RESULTS}

Figure 1 shows the probability of e-cigarette use as a function of developmental age by sex. This curve shows the age-varying intercept transformed from the logit to the probability scale, reflecting the overall

Figure 1. Probability of e-cigarette use by gender

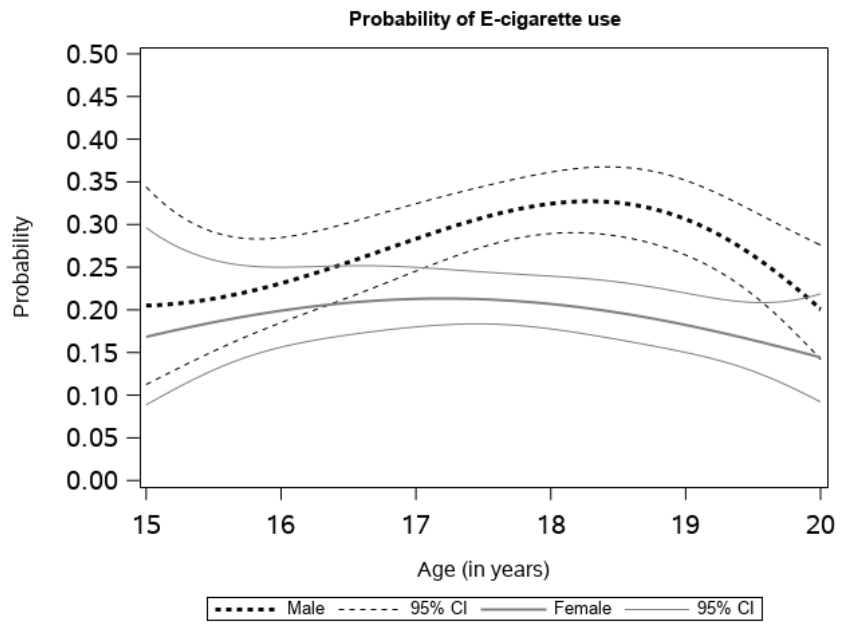

rate of e-cigarette use as a continuous function of age from 15 to 20 years. The probability of use for females was 0.17 (95\% CI: 0.09-0.30) at age 15 years, rising slowly to a high of 0.21 (95\% CI: 0.18-0.25) before gradually declining to 0.14 (95\% CI: $0.09-0.22)$ at age 20 years. For males, the probability of e-cigarette use at age 15 years was 0.21 (95\% CI: 0.11-0.34), followed by a gradual rise to a high of 0.33 (95\% CI: $0.29-0.37)$ at about age 18 years and then decreasing to 0.20 (95\% CI: $0.14-0.28)$ at age 20 years. The probabilities of e-cigarette use for males and females were significantly different after age 17 to 19.5 years, indicated by the non-overlapping confidence intervals.

\section{Peer use of e-cigarettes}

Figure 2 presents the age-varying association between peer e-cigarette use and current e-cigarette use. As associations are presented as odds ratios, confidence intervals not containing 1 (reference line included in plot) indicate a statistically significant association. For male students, the association was not statistically significant before age 16 years, while the association was statistically significant and stronger for females before age 16 years. At age 16 years, increasing the number of friends that use e-cigarettes by one is associated with higher odds of e-cigarette use of 1.39 (95\% CI: 1.05-1.85) for males and 2.01 (95\% CI: 1.51-2.68) for females. Between the ages of 16 and 20 years, this association was stable and statistically significant for both males and females, showing a similar pattern for both sexes. At age 20 years, the odds of e-cigarette use for each friend that uses

Figure 2. Association of peer e-cigarette use and current e-cigarette use

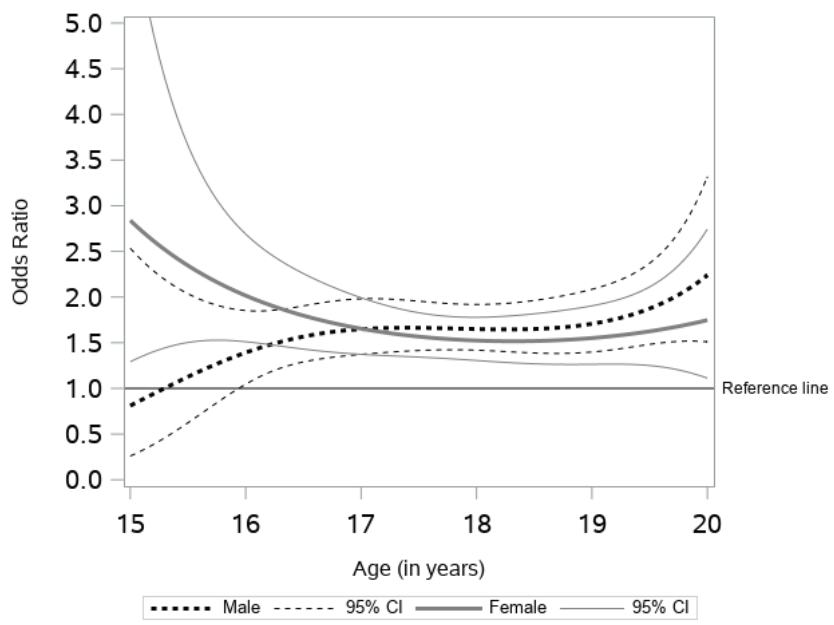


e-cigarettes was 2.24 (95\% CI: 1.51-3.32) for males and 1.74 (95\% CI: 1.11-2.74) for females.

\section{Exposure to e-cigarette commercials}

Figure 3 shows the age-varying association between exposure to e-cigarette commercials and current e-cigarette use. The age-varying effect model revealed that there was no statistically significant association between exposure and the current use of e-cigarettes among female participants of all ages. However, for male participants, the relationship is statistically significant between the age of 16 and 18 years. At 17 years, the odds of e-cigarette use for every unit increase in the frequency of exposure to e-cigarette commercials was 1.27 (95\% CI: 1.11-1.27). This suggests a positive association between the frequency of exposure to e-cigarette commercials and e-cigarette use for males between the ages of 16 and 18 years.

\section{Household e-cigarette use}

Figure 4 presents the age-varying association between household e-cigarette use and current e-cigarette use. For males, the odds ratio of e-cigarette use decreases from age 15 years, reaches a low point at age 17 years, gradually increases to its peak at age 18 years, and then is followed by another wave of similar up and down fluctuation with the bottom of the curve at age 19 years. The statistical significance is sustained only during the segment of the curve between ages 17 to 19 years. The odds ratio of e-cigarette use was 2.07 (95\% CI: $1.04-4.13)$ at age 17 years and 4.70 (95\% CI: 2.28-9.57) at age 18 years. The association was

Figure 3. Association between e-cigarette commercials and current e-cigarette use

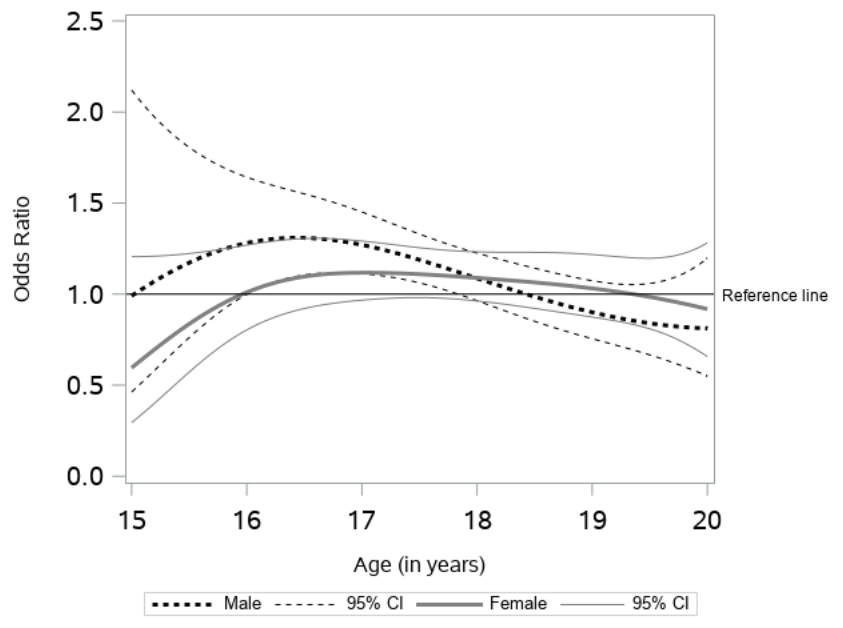

Figure 4. Association between household e cigarette use and current use

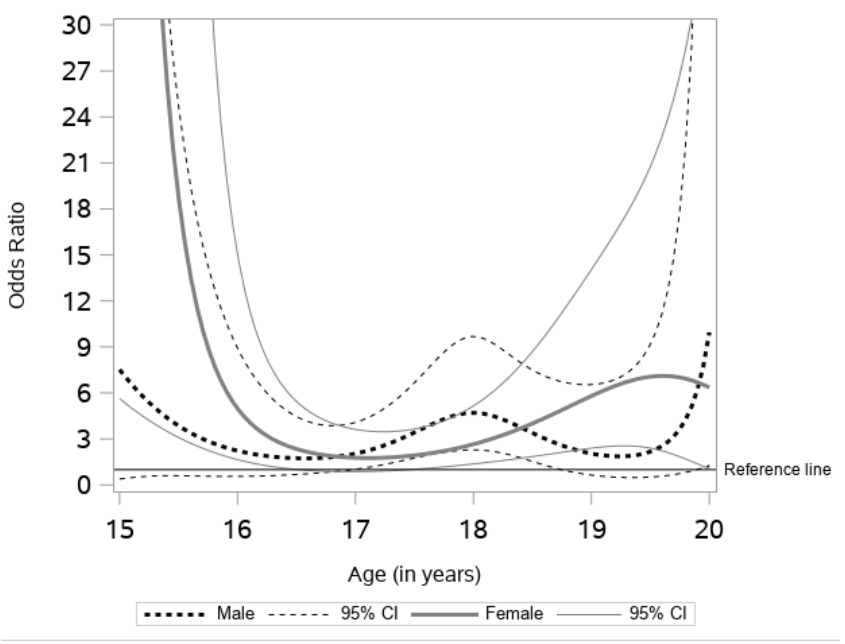

statistically significant for females up to 16.5 years and after 18 years. Female students in these age groups that reported living with an e-cigarette user were more likely to currently use e-cigarettes compared to those that did not live with an e-cigarette user, with odds ratio of 4.21 (95\% CI: $1.45-12.72)$ at age 16 years and 2.72 (95\% CI: $1.39-5.30)$ at age 18 years.

\section{DISCUSSION}

This study contributes to an understanding of the time-varying effects of several e-cigarette risk factors among alternative high school students. Our data suggest that the effect of such risk factors may be dynamic, not static, across adolescence to early adulthood in this sample. We found similar patterns of e-cigarette use among males and females until the age of 17 years, while after that age males had significantly higher rates of e-cigarette use. For both males and females, having friends that used e-cigarettes was associated with higher odds of current e-cigarette use. While the association remained stable for males and females between ages 16 to 20 years, the strongest association for females was before age 16 years and for males at age 20 years. The age-varying association between exposure to e-cigarette commercials was significant for males only; specifically, between the ages of 16 and 18 years. The association between household use and current use of e-cigarettes was positive across age, indicating higher odds of current use. For males, the association was significant between the ages of 17 and 19 years, while for females it was 
significant before 16.5 years and after 18 years.

Our findings indicate that peer e-cigarette use is an important risk factor for current e-cigarette use across the studied age groups and should be used as a potential intervention tool to reduce the increasing rate of e-cigarette use among at-risk adolescents. Specifically, our results suggest that having a friend that uses e-cigarettes is associated with higher odds of e-cigarette use across all ages for females and for males over the age of 16 years. This is consistent with previous studies on e-cigarette use and tobacco smoking that show the impact of peers on adolescent use $^{30-32}$. Interestingly, the association was strongest for females before 16 years and strongest for males at age 20 years, highlighting two potential stages of development where intervention could be targeted. In addition, the model also suggests that the influence of close friends from adolescent years may continue into adulthood. Studies of tobacco smoking have shown that two underlying processes explain the relationship-friend selection and peer influence ${ }^{33}$. The findings in this study lend further support to the effect of peer influence on current e-cigarette use among youths attending alternative high schools while demonstrating that this effect remains consistent throughout the adolescent years and possibly into adulthood.

Our analysis of e-cigarette commercials suggests that the frequency of exposure to e-cigarette commercials was associated with significantly higher odds of e-cigarette use for males only and specifically between the ages of 16 and 18 years. Previous studies have documented a positive association between exposure to commercials and advertisement of e-cigarettes and the likelihood of using e-cigarettes ${ }^{20,22,34,35}$. However, none of these studies examined the age at which the relationship may be significant, nor did they examine the associations by sex. Adolescents are frequent users of the internet and are highly receptive to advertising. Therefore, the high amount of youth-appealing content in e-cigarette commercials ${ }^{36,37}$, along with frequent exposure to e-cigarette commercials, may increase adolescents' susceptibility and acceptability of e-cigarettes-increasing the likelihood of e-cigarette use and eventually tobacco cigarette use ${ }^{19,34}$. Given the observed association between reported exposure to e-cigarette commercials and e-cigarette use from our study, future studies should investigate the underlying process or mechanism of this association. Also, future intervention studies are needed that examine if limiting exposure to e-cigarette commercials may be a useful tool in decreasing e-cigarette use in this specific population.

Contrary to our findings, previous studies observed stronger associations when examining advertising and the use of or susceptibility to the use of e-cigarettes ${ }^{20,35,38}$. The lower effect and lack of significance in this study may be due to the measure of e-cigarette commercial exposure used. Two initial questions asked participants if they had ever seen an e-cigarette commercial on television and the internet. Due to these questions, participants in our study may have indicated only the frequency of exposure from television and internet sources. Studies of traditional middle and high school students suggest that retail stores may be the main medium of exposure, with internet, television and print media being a significant source of exposure as well ${ }^{19}$. Thus, the magnitude of the association between e-cigarette advertising exposure and current e-cigarette use may be underestimated in this analysis.

Previous studies have found an association between household e-cigarette use and current use of e-cigarettes ${ }^{15,16}$. One study found that e-cigarette use by other household members may increase, by up to four times, the likelihood of current use of e-cigarettes ${ }^{16}$. Our findings support this reasoning, observing positive associations between exposure to household use of e-cigarettes and current use. Adolescents who have a family member using e-cigarettes in the home may obtain the device from the family member ${ }^{32}$. In addition, having a household member that uses e-cigarettes may provide easy access to e-cigarette devices, which may contribute to adolescent use.

Similar to peer use and exposure to e-cigarette commercials, the age-varying association between household use and the current use of e-cigarettes differed by males and females. While household use was associated with significantly higher odds of e-cigarette use between the ages of 17 and 19 years for males, it was associated with current use before 16.5 years and after 18 years, for females.

\section{Strengths and limitations}

The findings of this study must be considered along 
with its limitations. First, as previously stated, because of the initial questions asking about exposure to e-cigarette commercials on television or the internet, participants may have left out frequency of exposure from retail stores, print media, billboards and other important sources besides television and the internet. This may have resulted in under-reporting of exposure to e-cigarette commercials in this sample. Second, due to the cross-sectional nature of the data, causal inferences cannot be made with confidence from the observed associations. On the association between peer use and current use, this study is unable to determine the direction of the association. It is likely that e-cigarette users were more likely to select friends who also used e-cigarettes. Similarly, e-cigarette users may have a more heightened sensitivity to e-cigarette advertising, making them more likely to recall seeing a commercial. Third, this study relies on self-reported information by adolescents, which may be subject to biases such as social desirability and recall bias. Fourth, the generalizability of the findings in this study is limited by the low response rate, only $15.4 \%$ of the alternative high school students that were contacted completed the survey. Finally, because the data from the first wave were collected in 2015 , e-cigarette products, advertising and social norms regarding e-cigarettes are likely to have changed for adolescents in the intervening period. Future studies may examine if the findings in this study have changed as well.

Despite these limitations, the study provides new insight into the relationship between current e-cigarette use and risk factors across age among alternative high school students. Further, this study identifies potentially vulnerable developmental stages for male and female adolescents that may be targetable for future interventions. Future research could follow in this direction to further examine the effect of specific influences such as separating TV and internet sources of commercials, breaking down internet sources into types of social media and other online sources, and differentiating influences from different household members.

\section{CONCLUSIONS}

E-cigarettes are now the most commonly used tobacco product among adolescents and young adults in the $\mathrm{US}^{1,11}$. Our findings further support the current evidence that environmental factors, such as advertising and both social and household environment, are important predictors of current e-cigarette use. Specifically, exposure to e-cigarette advertising, use by peers and use by household members were positively associated with current use among adolescents. The association was age-varying and differed for males and females. As the rate of e-cigarette use among adolescents and young adults in the US increases, peer influence may contribute to an exponential increase in e-cigarette use. Additionally, more research focusing on the social dynamics of e-cigarette use is needed to curb the growing trend.

\section{REFERENCES}

1. U.S. Department of Health and Human Services. E-Cigarette Use Among Youth and Young Adults: A Report of the Surgeon General. Atlanta, GA: U.S. Department of Health and Human Services, Centers for Disease Control and Prevention, National Center for Chronic Disease Prevention and Health Promotion, Office on Smoking and Health; 2016.

2. Cullen K, Ambrose B, Gentzke A, Apelberg B, Jamal A, King B. Notes from the Field: Use of Electronic Cigarettes and Any Tobacco Product Among Middle and High School Students — United States, 2011-2018. MMWR Morb Mortal Wkly Rep. 2018;67. doi:10.15585/mmwr.mm6745a5

3. Pisinger C, Døssing M. A systematic review of health effects of electronic cigarettes. Prev Med. 2014;69:248260. doi:10.1016/j.ypmed.2014.10.009

4. Soneji S, Barrington-Trimis JL, Wills TA, et al. Association Between Initial Use of e-Cigarettes and Subsequent Cigarette Smoking Among Adolescents and Young Adults: A Systematic Review and Meta-analysis. JAMA Pediatr. 2017;171(8):788797. doi:10.1001/jamapediatrics.2017.1488

5. U.S. Department of Health and Human Services. The Health Consequences of Smoking -- 50 Years of progress: A Report of the Surgeon General. Atlanta, GA: U.S. Department of Health and Human Services, Centers for Disease Control and Prevention, National Center for Chronic Disease Prevention and Health Promotion, Office on Smoking and Health; 2014.

6. Sussman S, Stacy AW, Dent CW, et al. Continuation high schools: Youth at risk for drug abuse. J Drug Educ. 1995;25(3):191-209. doi:10.2190/hdqh-xd21-gjt0-9g8v

7. Grunbaum JA, Basen-Engquist K. Comparison of Health Risk Behaviors Between Students in a Regular High School and Students in an Alternative High School. J Sch Health. 1993;63(10):421-425. doi:10.1111/j.1746-1561.1993.tb06072.x

8. Grunbaum JA, Kann L, Kinchen SA, et al. Youth Risk Behavior Surveillance--National Alternative High School Risk Behavior Survey, United States, 1998. MMWR 
Surveillance Summaries. 1999;48. https://www.cdc.gov/ mmwr/preview/mmwrhtml/ss4807a1.htm\#top. Accessed July 30, 2019.

9. Johnson KE, McMorris BJ, Kubik MY. Comparison of health-risk behaviors among students attending alternative and traditional high schools in Minnesota. J Sch Nurs. 2013;29(5):343-352. doi:10.1177/1059840512469409

10. Grunbaum JA, Lowry R, Kann L. Prevalence of healthrelated behaviors among alternative high school students as compared with students attending regular high schools. J Adolesc Health. 2001;29(5):337-343. doi:10.1016/s1054-139x(01)00304-4

11. Gentzke AS, Creamer M, Cullen KA, et al. Vital Signs: Tobacco Product Use Among Middle and High School Students-United States, 2011-2018. MMWR Morb Mortal Wkly Rep. 2019;68(6):157. doi:10.15585/mmwr.mm6806e1

12. Oetting ER, Donnermeyer JF. Primary socialization theory: the etiology of drug use and deviance. I. Subst Use Misuse. 1998;33(4):995-1026. doi:10.3109/10826089809056252

13. Catalano RF, Hawkins JD. The social development model: A theory of antisocial behavior. In: Delinquency and crime: Current theories. Cambridge University Press; 1996: 149.

14. Morello P, Pérez A, Peña L, et al. Prevalence and predictors of e-cigarette trial among adolescents in Argentina. Tob Prev Cessat. 2016;2(December). doi:10.18332/tpc/66950

15. Barrington-Trimis JL, Berhane K, Unger JB, et al. The E-cigarette Social Environment, E-cigarette Use, and Susceptibility to Cigarette Smoking. J Adolesc Health. 2016;59(1):75-80. doi:10.1016/j.jadohealth.2016.03.019

16. Barrington-Trimis JL, Berhane K, Unger JB, et al. Psychosocial Factors Associated With Adolescent Electronic Cigarette and Cigarette Use. Pediatrics. 2015;136(2):308-317. doi:10.1542/peds.2015-0639

17. Hwang JH, Park SW. Association between Peer Cigarette Smoking and Electronic Cigarette Smoking among Adolescent Nonsmokers: A National Representative Survey. PLoS One. 2016;11(10). doi:10.1371/journal.pone.0162557

18. Liao Y, Huang Z, Huh J, Pentz MA, Chou CP. Changes in Friends' and Parental Influences on Cigarette Smoking From Early Through Late Adolescence. J Adolesc Health. 2013;53(1):132-138. doi:10.1016/j.jadohealth.2013.01.020

19. Marynak K, Gentzke A, Wang TW, Neff L, King BA. Exposure to electronic cigarette advertising among middle and high school students-United States, 20142016. MMWR Morb Mortal Wkly Rep. 2018;67(10):294. doi:10.15585/mmwr.mm6710a3

20. Mantey DS, Cooper MR, Clendennen SL, Pasch KE, Perry CL. E-Cigarette Marketing Exposure Is Associated With E-Gigarette Use Among U.S. Youth. J Adolesc Health. 2016;58(6):686-690. doi:10.1016/j.jadohealth.2016.03.003

21. Leventhal AM, Strong DR, Kirkpatrick MG, et al. Association of electronic cigarette use with initiation of combustible tobacco product smoking in early adolescence. J Am Med Assoc. 2015;314(7):700-707. doi:10.1001/jama.2015.8950

22. Villanti AC, Rath JM, Williams VF, et al. Impact of Exposure to Electronic Cigarette Advertising on Susceptibility and Trial of Electronic Cigarettes and Cigarettes in US Young Adults: A Randomized Controlled Trial. Nicotine Tob Res. 2016;18(5):1331-1339. doi:10.1093/ntr/ntv235

23. Beleva Y, Pike JR, Miller S, Xie B, Ames SL, Stacy AW. Share of advertising voice at the point-of-sale and its influence on at-risk students' use of alternative tobacco products. Nicotine Tob Res. 2018. doi:10.1093/ntr/nty152

24. Miller S, Pike J, Stacy AW, Xie B, Ames SL. Negative affect in at-risk youth: Outcome expectancies mediate relations with both regular and electronic cigarette use. Psychol Addict Behav. 2017;31(4):457-464. doi:10.1037/adb0000272

25. Shiyko M, Naab P, Shiffman S, Li R. Modeling Complexity of EMA Data: Time-Varying Lagged Effects of Negative Affect on Smoking Urges for Subgroups of Nicotine Addiction. Nicotine Tob Res. 2014;16(Suppl 2):S144-S150. doi:10.1093/ntr/ntt109

26. Li R, Dziak JJ, Tan X, Huang L, Wagner AT, Yang J. TVEM (time-varying effect model) SAS macro users' guide (Version 3.1.1). https://www.methodology.psu. edu/files/2019/03/TVEM_3.1.1-1fxcco8.pdf. Published 2017. Accessed July 30, 2019.

27. Lanza ST, Vasilenko SA, Russell MA. Time-Varying Effect Modeling to Address New Questions in Behavioral Research: Examples in Marijuana Use. Psychol Addict Behav. 2016;30(8):939-954. doi:10.1037/adb0000208

28. Tan X, Shiyko MP, Li R, Li Y, Dierker L. A time-varying effect model for intensive longitudinal data. Psychol Methods. 2012;17(1):61-77. doi:10.1037/a0025814

29. Shiyko MP, Lanza ST, Tan X, Li R, Shiffman S. Using the Time-Varying Effect Model (TVEM) to Examine Dynamic Associations between Negative Affect and Self Confidence on Smoking Urges: Differences between Successful Quitters and Relapsers. Prev Sci. 2012;13(3):288-299. doi:10.1007/s11121-011-0264-z

30. Kaleta D, Wojtysiak P, Polańska K. Use of electronic cigarettes among secondary and high school students from a socially disadvantaged rural area in Poland. BMC Public Health. 2016;16. doi:10.1186/s12889-016-3417-y

31. Perikleous EP, Steiropoulos P, Paraskakis E, Constantinidis TC, Nena E. E-Cigarette Use Among Adolescents: An Overview of the Literature and Future Perspectives. Front Public Health. 2018;6. doi:10.3389/fpubh.2018.00086

32. Vogel EA, Ramo DE, Rubinstein ML. Prevalence and correlates of adolescents' e-cigarette use frequency and dependence. Drug Alcohol Depend. 2018;188:109-112. doi:10.1016/j.drugalcdep.2018.03.051

33. Schaefer DR, Haas SA, Bishop NJ. A dynamic model of U.S. adolescents' smoking and friendship 
networks. Am J Public Health. 2012;102(6):e12-e18. doi:10.2105/AJPH.2012.300705

34. Agaku IT, Ayo-Yusuf OA. The Effect of Exposure to Pro-Tobacco Advertising on Experimentation With Emerging Tobacco Products Among U.S. Adolescents. Health Educ Behav. 2014;41(3):275-280. doi:10.1177/1090198113511817

35. Pierce JP, Sargent JD, Portnoy DB, et al. Association Between Receptivity to Tobacco Advertising and Progression to Tobacco Use in Youth and Young Adults in the PATH Study. JAMA Pediatr. 2018;172(5):444-451. doi:10.1001/jamapediatrics.2017.5756

36. Padon AA, Maloney EK, Cappella JN. Youth-Targeted E-cigarette Marketing in the U.S. Tob Regul Sci. 2017;3(1):95-101. doi:10.18001/TRS.3.1.9

37. Padon AA, Lochbuehler K, Maloney EK, Cappella JN. A Randomized Trial of the Effect of Youth Appealing E-Cigarette Advertising on Susceptibility to Use E-Cigarettes Among Youth. Nicotine Tob Res. 2018;20(8):954-961. doi:10.1093/ntr/ntx155

38. Singh T, Agaku IT, Arrazola RA, et al. Exposure to Advertisements and Electronic Cigarette Use Among U.S. Middle and High School Students. Pediatrics. 2016;137(5). doi:10.1542/peds.2015-4155

ACKNOWLEDGEMENTS

The authors wish to thank Alan Stacy for guidance and feedback during the process of developing this manuscript. We thank Sandy Asad, Sara J. Asad, Melissa Garrido, Sarah Z. Gonzalez, Hannah Jornacion, and Brenda Lisa Lucero for their tireless efforts recruiting and tracking alternative high school students.

CONFLICTS OF INTEREST

The authors have completed and submitted the ICMJE Form for Disclosure of Potential Conflicts of Interest and none was reported.

\section{FUNDING}

This work was supported by the National Institute of Child Health and Human Development and the Food and Drug Administration Center for Tobacco Products (R01HD077560). The content is solely the responsibility of the authors and does not necessarily represent the official views of the National Institutes of Health or the Food and Drug Administration.

PROVENANCE AND PEER REVIEW

Not commissioned; externally peer reviewed. 